Proc. Indian Acad. Sci. (Earth Planet. Sci.), Vol. 102, No. 4, December 1993, pp. 507-519.

(C) Printed in India.

\title{
Impact of moisture variations on the circulation of the south-west monsoon
}

\author{
C M KISHTAWAL ${ }^{1}$, P K PAL ${ }^{1}$, M S NARAYANAN ${ }^{1}$, \\ S K MANNA ${ }^{2}$, O P SHARMA ${ }^{3}$, SANGEETA AGARWAL ${ }^{3}$ and \\ H C UPADHYAYA ${ }^{3}$ \\ ${ }^{1}$ Meteorology and Oceanography Division, Space Applications Centre, Ahmedabad 380053, \\ India \\ ${ }^{2}$ Department of Physics, University of Poona, Pune 411007 \\ ${ }^{3}$ Centre for Atmospheric Sciences, Indian Institute of Technology, Delhi 110016
}

MS received 28 April 1993; revised 5 November 1993

\begin{abstract}
Abatract. The impact of moisture anomalies on the circulation of the south-west Indian monsoon has been studied with a general circulation model. Newtonian relaxation is adopted to subject the model atmosphere under sustained moisture anomalies. The impact of negative anomalies of moisture was seen as a divergent circulation anomaly, while the positive anomaly was a stronger convergent anomaly. Although the humidity fields display a resilient behaviour, and relax back to normal patterns 1-2 days after the forcing terms in humidity are withdrawn, the circulation anomalies created by the moisture variation keeps growing. A feedback between positive moisture anomalies and low level convergence exists, which is terminated in the absence of external forcings.
\end{abstract}

Keyworts. LMD-GCM; nudging moisture anomalies.

\section{Introduction}

Proper specification of atmospheric moisture fields in the initial condition is of importance for numerical weather prediction over the tropics (Krishnamurti and Kanamitsu 1973). Smagorinsky et al (1970) showed that in extratropical regions, the models are capable of generating their own humidity fields with reasonable accuracy, and humidity may be considered a redundant parameter in these regions. However, over the tropics no simple relationship between mass and wind fields exists, and it takes a longer time to establish mutual adjustment between the two, and to generate vertical velocity and moisture fields. This aspect makes it necessary that the humidity fields be defined in the initial states of the model through accurate observations.

The scope of this paper is to present the impact of moisture anomalies on the monsoon circulation on a medium range time scale ( 3 days) using a general circulation model. The anomaly may be viewed as a systematic bias in observations, or it may be considered as an abnormal atmospheric state. The next section briefly describes the model and section 3 describes the experiments. In section 4, the interaction of moisture with the monsoon circulation is analysed. Section 5 concludes the study. 


\section{The monsoon general circulation model}

The model used for the present study is the general circulation model of Laboratorie de Meteorologi Dynamique (LMD) Paris, France, which was modified by IIT, Delhi for monsoonal studies (Sharma et al 1986). It has 64 zonal and 50 meridional grid points. To improve the resolution in some specific area of interest, the model is implemented with a stretching function in longitudinal direction as follows:

$$
\lambda=\lambda_{0}+x-\frac{1}{2} \sin (x) ; \phi=\sin ^{-1}(y)
$$

$\lambda$ and $\phi$ are the longitude and latitude respectively. For our studies we have fixed $\lambda=70^{\circ} \mathrm{E}$. The above function contracts the mesh by a factor of 2 at $\lambda=\lambda_{0}$ and stretches it by a factor of 1.5 at $\lambda=\lambda_{0}+180^{\circ}$. The meridional gridsize is $225 \mathrm{~km}$ at the equator and zonal resolution varies from $315 \mathrm{~km}$ at $\lambda=70^{\circ} \mathrm{E}$ to $945 \mathrm{~km}$ at $\lambda=110^{\circ} \mathrm{W}$. In the vertical, there are eleven unevenly spaced sigma levels $(\sigma=1 \cdot 0$, $0.979,0.941,0.873,0.770,0.638,0.489,0.340,0.208,0 \cdot 106,0.038$ and 0.000). An Arakawa C-grid is employed for space differencing while time integration uses the leap frog scheme, except for the initial 12 hours when Matsuno's double time step integration is employed to damp high frequency gravity waves.

One of the problems of stretched grid approach is that the advection of small scale structures by a uniform velocity out of the high resolution area is not allowed (Courtier and Geleyn 1988). This leads to the trapping of the small scale energy in the high resolution area as in the limited area models, if appropriate dissipation is not added. Our experience shows that the use of an efficient local dissipation makes the problem of energy trapping less serious. There is also a possibility of some distortion in the output (Courtier and Geleyn 1988).

Vertical turbulent fluxes are parameterised using the vertical eddy diffusion approximation, where the mixing length scale is taken from Smagorinsky et al (1965). Moist convective adjustment takes place when the air is supersaturated and the adjustment scheme is similar to Manabe and Strickler (1965). Kuo's (1965) parameterisation is used for cumulus convection. For parameterisation of solar radiation, the transmission functions for water vapour, ozone and carbon dioxide are calculated from the LOWTRAN 3-b model (Selby et al 1976).

\section{Moisture experiments}

The model integration was started with FGGE-III (B) analysed fields of 11 June 1979, interpolated to model grid points. This belongs to the onset phase of the 1979 monsoon. To assess the impact of moisture variability over the Arabian Sea on monsoon circulation, two experiments are made. In the first experiment, the moisture is subjected to sustained negative anomalies over the Arabian Sea, while in the second experiment the anomalies are made positive keeping the relative humidity always less than $90 \%$. The amplitude of moisture fluctuation is of a similar order as deduced from observations (Cadet 1986; Kishtawal et al 1991). To ensure a slow rate of change of the moisture fields towards the anomaly fields, the moisture fields are modified to the target values by means of nudging. Figure 1(a) shows the domain where the 


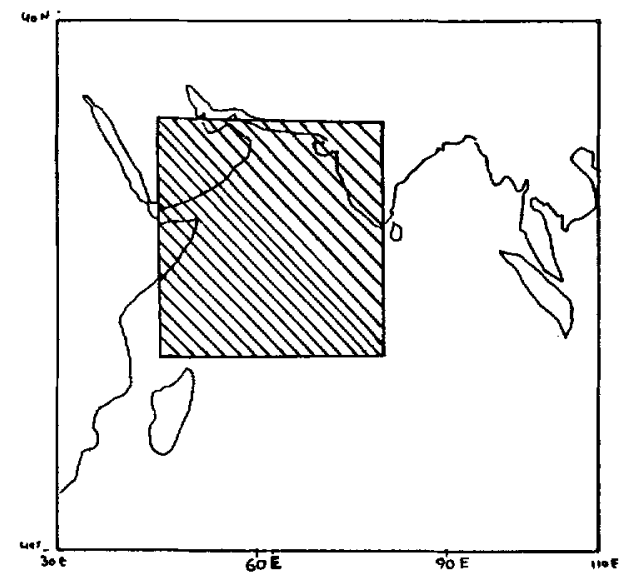

(a)

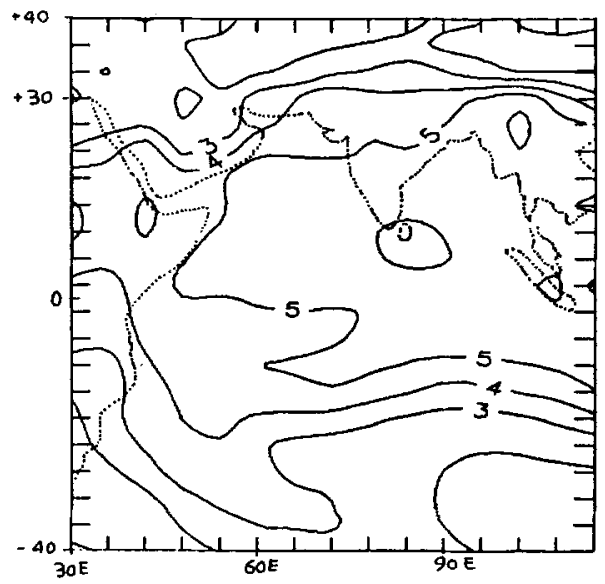

(b)

Figure 1. (a) Shaded region identifies the experimental domain. (b) Vertically integrated moisture on day-1 of control run (unit: $\mathrm{gm} / \mathrm{cm}$ ).

moisture variations have been carried out. Nudging or Newtonian relaxation (Hoke and Anthes 1976; Ramamurthy and Carr 1987) allows one to assimilate the observed fields with model forecast fields smoothly. The moisture equation is expressed in the model by

$$
\frac{\partial Q}{\partial t}=F(g, \hat{x}, t)+\varepsilon G(\hat{x}, t)\left(Q_{0}-Q\right)
$$

All physical processes, such as, advection and other source and sink terms are included in $F(g, \hat{x}, t)$, where $g$ denotes the independent variables, $\hat{x}$ represents the independent spatial variables and $t$ is time. $\varepsilon$ represents the weight matrix, which is the unit matrix here. $Q_{0}$ represents the target fields. The values of $Q_{0}$ are generated at each time step by linear interpolation between model fields at $0 \mathrm{~h}$ and simulated fields at $24 \mathrm{~h}$. The magnitude of the relaxation coefficient $G(g, t)$ determines the importance of the nudging term relative to the internal dynamic coupling implied by other terms in the model equation. 


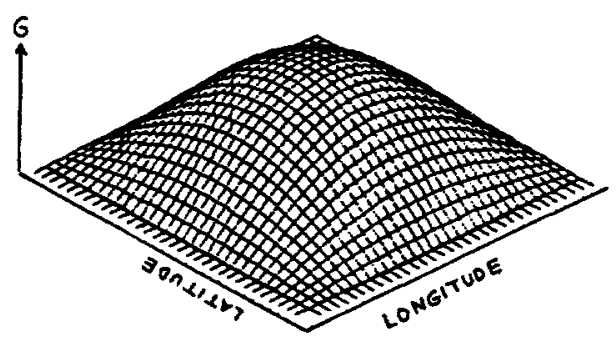

(a)

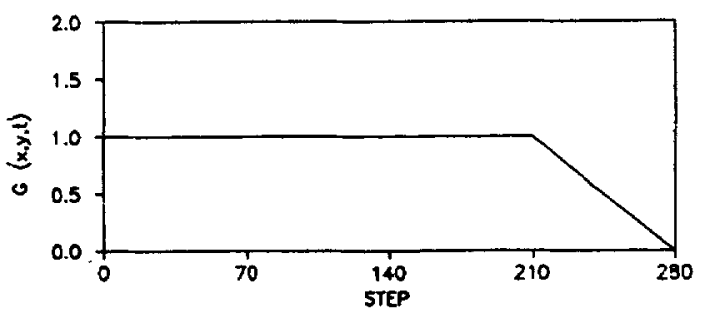

(b)

Figure 2 (a) Spatial and (b) temporal variation of the nudging coefficient $G(x, y, t)$.

Figure 1(b) shows the vertically integrated moisture fields on 12 June 1979 as predicted by the model. The model moisture fields were nudged from 11 June 1979 (day 0) towards 75 percent/125 percent of 12 June field (day 1) over the Arabian Sea $\left(30^{\circ} \mathrm{E}-80^{\circ} \mathrm{E}\right.$ and $\left.10^{\circ} \mathrm{S}-30^{\circ} \mathrm{N}\right)$ in experiment $1 /$ experiment 2 by using a region dependent nudging coefficient. The value of $G$ at the centre of nudging domain is $0.5 \times 10^{-3}$ which decreases to zero towards the edges of the domain following a smooth cosine taper variation and remains zero outside this domain (figure 2a). This choice of nudging coefficient allows a smooth transition of moisture fields to simulated values. The nudging coefficient remains constant at a particular grid point for 18 hours of integration after which it decreases linearly to zero in the next 6 hours (figure 2b).

The moisture anomalies were introduced by nudging to put the model under sustained moisture anomalies over a fixed period of time. Smagorinsky et al (1970) concluded from their studies, which we also verified with the present model, that any moisture anomaly introduced in the initial condition of the model gets 'washed off' after a certain time, say one day, and the model humidity fields always relax back to a normal pattern. This 'normal' pattern is defined by wind fields, model physics, particularly moist convective adjustment and cumulus parameterization. It may differ from the actual humidity patterns in the atmosphere. Moreover, though the humidity fields revert back to normal within 1-2 days, the circulation gets altered noticeably in this period. Through this study we want to assess the impact on circulation, if the model humidity fields are continuously deviated from their model defined patterns.

\section{Results and discussions}

\subsection{Control run}

The control run is the three day integration of GCM starting from 11 June 1979. No forcing or nudging terms are included in the model equations during the control run. 
(a)

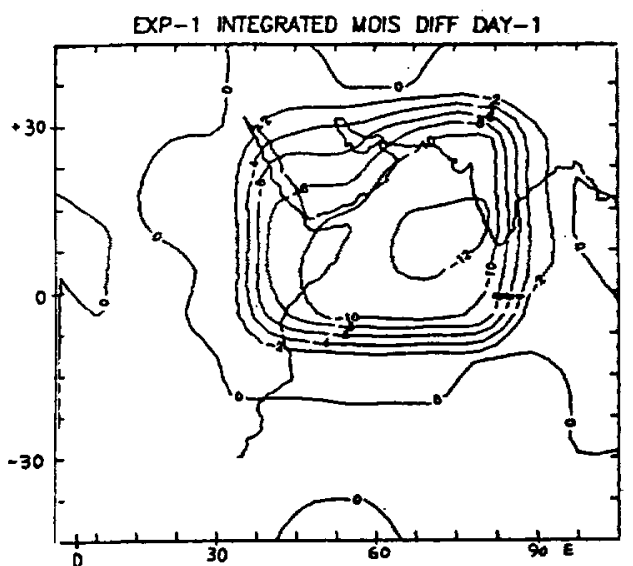

(c)

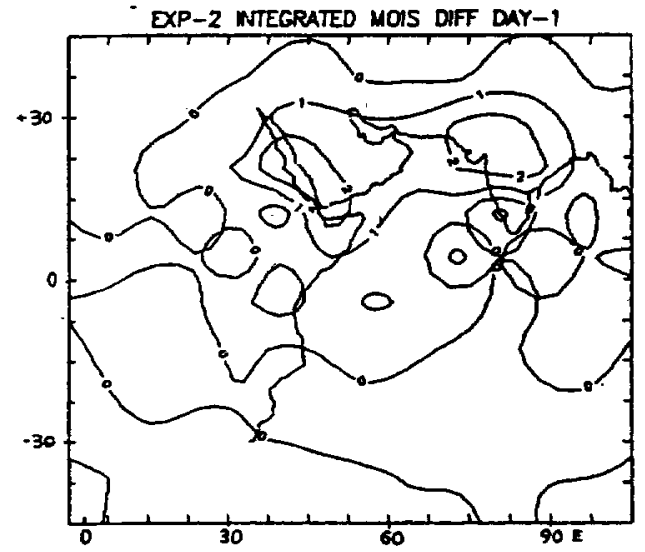

(b)

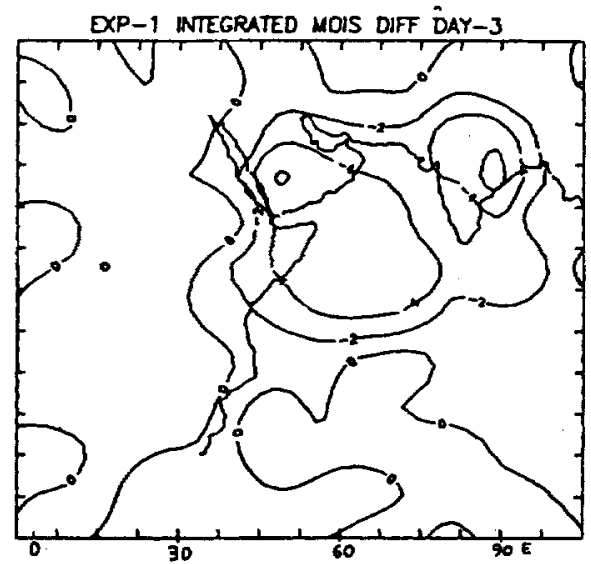

(d)

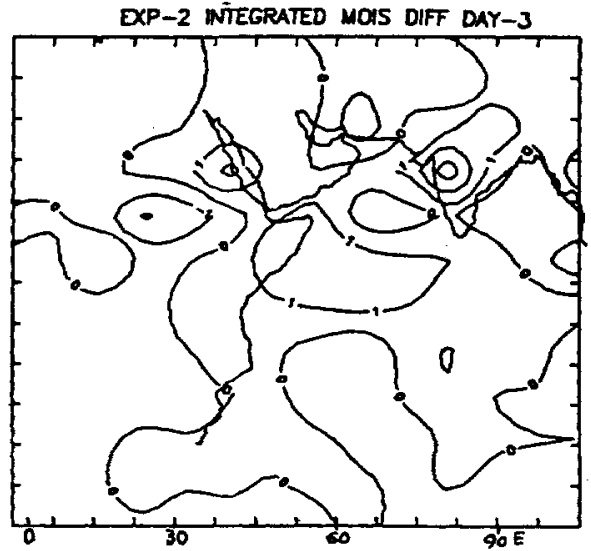

Figure 3. Vertically integrated moisture field differences (Experiment minus control). (a) Experiment-1 day-1 (b) Experiment-1 day-3 (c) Experiment-2, day-1 (d) Experiment-2, day-3. (Unit $=0.2 \mathrm{gm} / \mathrm{cm}^{2}$ ).

\section{2(a) Experiment 1}

In this experiment the moisture was decreased at all levels over the nudging domain by relaxing the initial day moisture fields to $75 \%$ of their day-1 values in the interval of one day. Figure 3 ( $a$ and $b$ ) shows the difference of vertically integrated moisture fields (experiment minus control) on day 1 and day 3 of integration. The effect of nudging led to a maximum change of vertically integrated moisture at $55 \mathrm{E} 15 \mathrm{~N}$, which was $-2.5 \mathrm{gm} / \mathrm{cm}^{2}$ and the minimum reduction took place at the edges of the domain. Levels below $700 \mathrm{mb}$ contributed to $80 \%$ of the total moisture difference while the remaining $20 \%$ came from the upper levels. On the initial day, the patterns of moisture difference were identical at both the upper and the lower layers. On day 3 the maximum difference of vertically integrated moisture field had shrunk to $-0.5 \mathrm{gm} / \mathrm{cm}^{2}$ at the lower layer, and there is a secondary negative maxima forming northeast of the primary maxima over the Indian peninsula. Interestingly, there is a consistent decrease in moisture at the upper layer over the Indian peninsula, while 


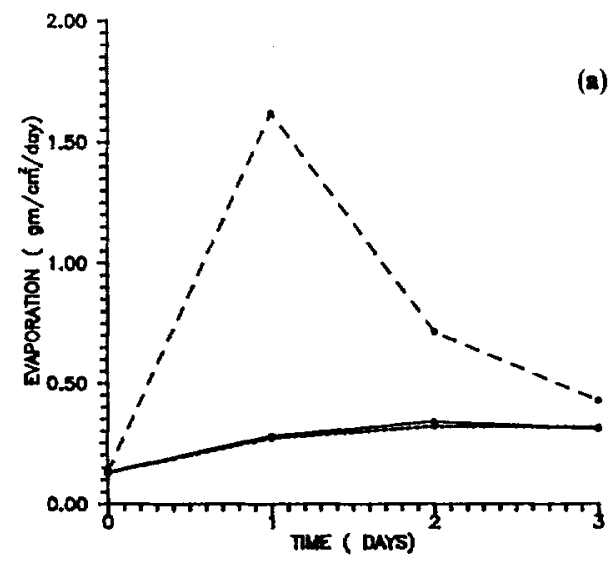

(a)

(b)

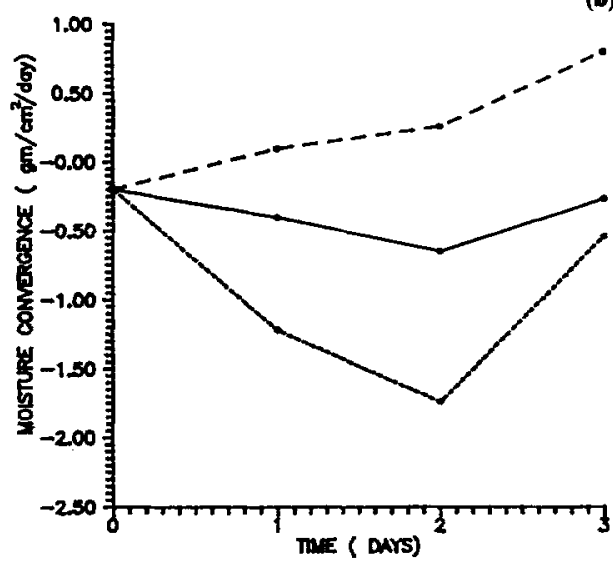

Figure 4. Time evolution of (a) Evaporative flux and (b) Moisture flux convergence for experiment-1 and experiment-2; _- control; --_ expt. $1 ; \cdots$ expt. 2.

at the lower layer, the moisture fields tend to get back to normal values at a fast rate, except over the Indian subcontinent where the rate of recovery is slow. It indicates the dominance of evaporative fluxes over advective fluxes or convergence under low saturation conditions, which is also indicated by figure 4 . This aspect is discussed separately in section 4.3 .

Figure 5 shows the difference of wind fields (experiment minus control) on day 1 and day 3 at 850 and $200 \mathrm{mb}$. A weak yet systematic divergence pattern seems to emerge over the nudging domain at $850 \mathrm{mb}$ on day 1 . The rms wind difference at this stage is $0.5 \mathrm{~m} / \mathrm{s}$. By the third day (figure $5 \mathrm{c}$ ) the difference fields are significantly stronger with the rms wind difference of $2.6 \mathrm{~m} / \mathrm{s}$. A comparison of the divergence difference fields indicates that the divergence patterns are not only stronger on day 3 , but they have moved north of their day-1 position. There is a development of convergence patterns to the east and northeast of the maximum divergence location. The circulation difference at $200 \mathrm{mb}$ is very weak on day 1 , with an $\mathrm{rms}$ wind difference of $0.5 \mathrm{~m} / \mathrm{s}$. Considering the fact that the mean wind speed at $200 \mathrm{mb}$ is at least three times larger than that at $850 \mathrm{mb}$, the circulation difference at $200 \mathrm{mb}$ is much weaker than at $850 \mathrm{mb}$ on day 1 . But, on subsequent days the difference wind fields at $200 \mathrm{mb}$ 
(a)

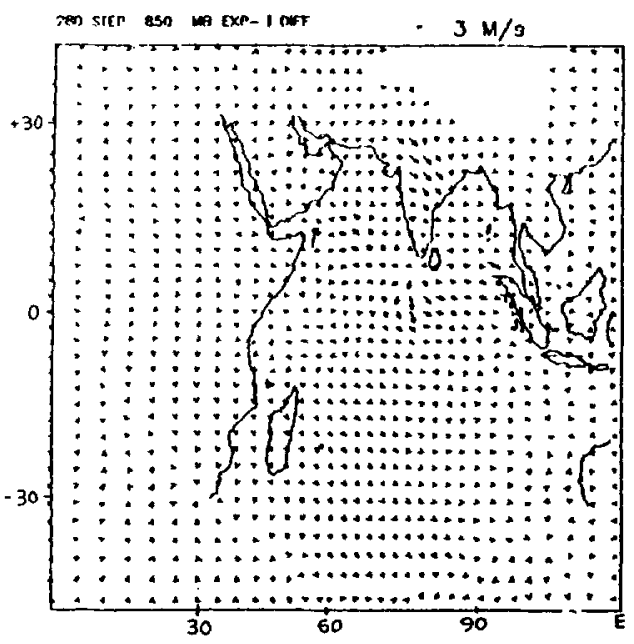

(c)

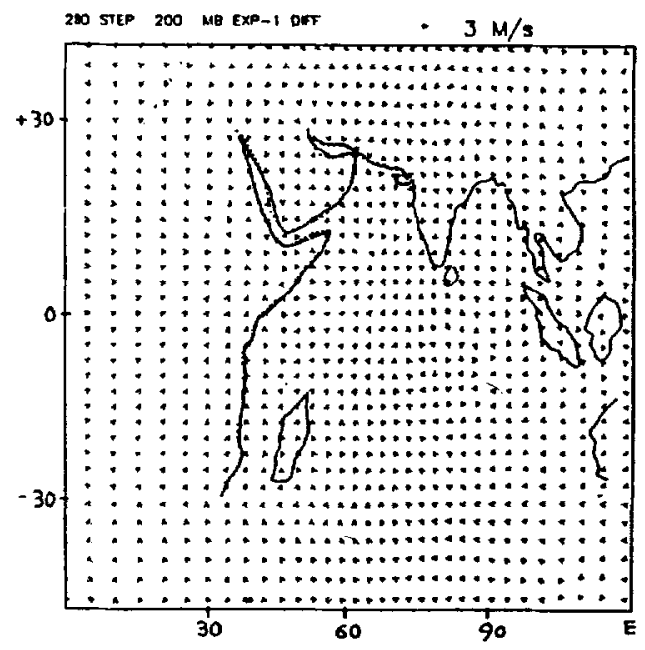

(b)

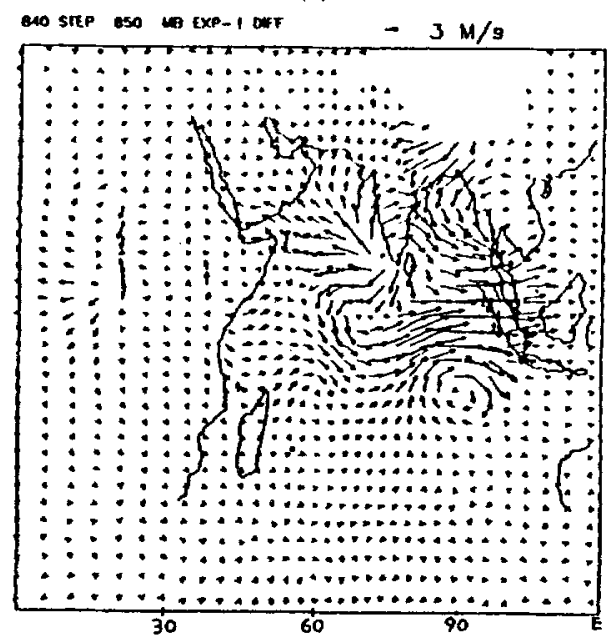

(d)

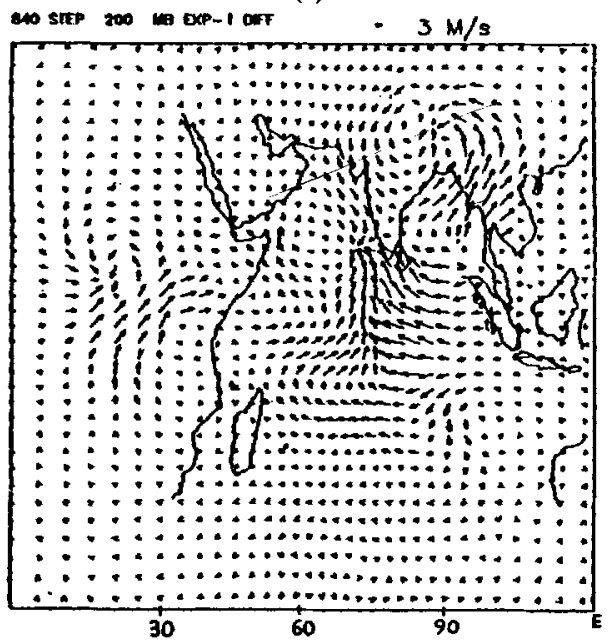

Figure 5. Wind difference vectors (Experiment minus control) for experiment 1 on (a) Day-1, $850 \mathrm{mb}$ (b) Day-1, $200 \mathrm{mb}$ (c) Day-3, $850 \mathrm{mb}$ and (d) Day-3, $200 \mathrm{mb}$ (similar vector length represents stronger wind at $200 \mathrm{mb}$ ).

become stronger than those at $850 \mathrm{mb}$ on day 3 with an $\mathrm{rms}$ wind difference of $4.3 \mathrm{~m} / \mathrm{s}$. A strong convergence pattern is formed over the east Arabian Sea, and another moderate one appears over the Tibetan region. The circulation difference at $200 \mathrm{mb}$ is a delayed response of circulation at lower levels. It seems that when the moist convective processes are weak or absent, the coupling between the lower and upper levels takes place on a longer time (almost one day) compared to a situation where moist processes actively modify the dynamics through latent heat release. This is indicated by the results of experiment- 2 discussed in the next section. 
(a)

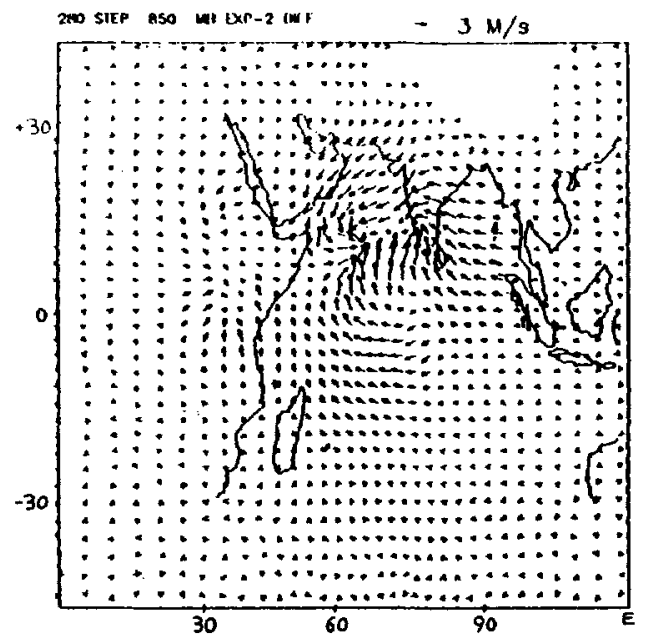

(c)

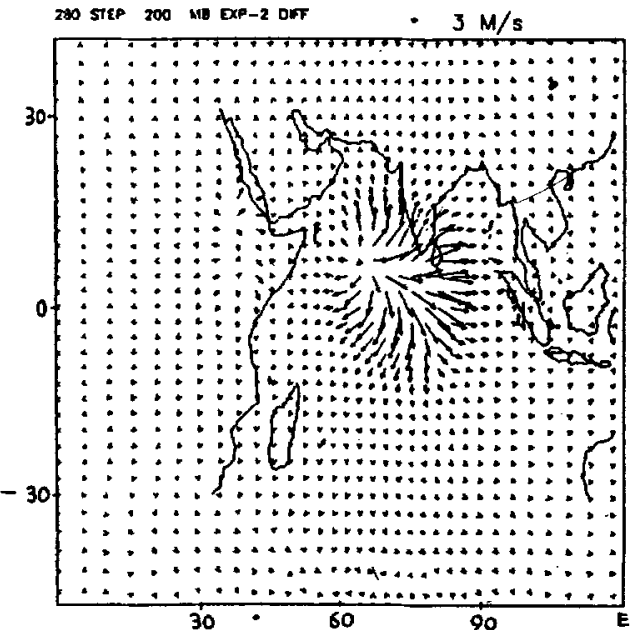

(b)

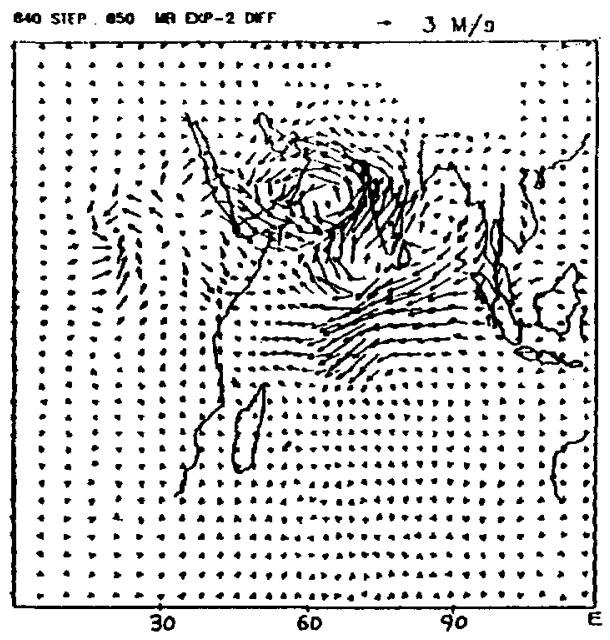

(d)

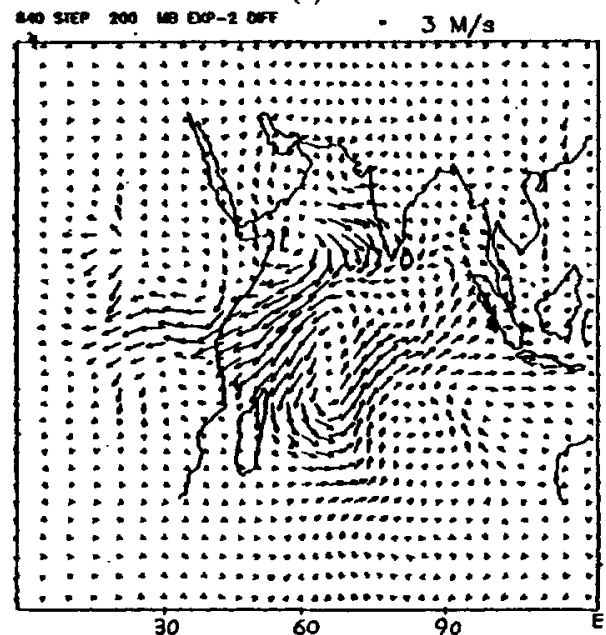

Figure 6. Same as figure 5 but for experiment-2.

\section{4:2(b) Experiment 2}

The set up for experiment 2 is similar to experiment 1 , but the moisture values are increased by relaxing the initial day moisture fields towards $125 \%$ of their day-1 values in the interval of one day, using the technique of nudging. The target moisture fields were not allowed to exceed $90 \%$ of their saturation values except at places where it already exceeds $90 \%$. In this process the near saturated points did not gain from nudging and, unlike experiment 1 , the moisture difference contours display an asymmetric pattern skewed towards the northeastern corner of nudging domain. Due to the constraint on the upper limit of moisture, the magnitude of the maximum moisture difference (experiment minus control) is only about one third as large as in experiment-1 on day 1 at the lower layer $\left(1 \mathrm{gm} / \mathrm{cm}^{2}\right)$ as in figure $3 \mathrm{c}$. The patterns of 
moisture difference at the upper layer are significantly different from experiment 1 . On day 1 the only significant difference at the upper layers occurs over the Indian subcontinent. On day 2, the differences in the lower layer are reduced by about $50 \%$ over the experimental domain, except over the northeastern region where the differences not only increase by about $25 \%$, but move eastward by about 1 grid length (about $300 \mathrm{~km}$ ). On day 3 , the differences are further reduced by $50 \%$ of day -2 values over most of the domain. But, over a few grid points inside the Indian subcontinent the differences continue to grow upto a maximum of $2 \mathrm{gm} / \mathrm{cm}^{2}$.

Above $700 \mathrm{mb}$, however, the differences grow consistently, i.e., the upper layer continues to gather more moisture on subsequent days. The increase of moisture in upper layers and in the northeastern sectors of the lower layers indicates the role of advection accompanied by lower level convergence in a saturated or supersaturated atmosphere.

The wind difference fields on day 1 indicate patterns of convergence at $850 \mathrm{mb}$ and divergence at $200 \mathrm{mb}$ which are strongest slightly to the northwest of the centre of the nudging region (figure 6). Table 1 shows the rms differences in wind speed at 850 and $200 \mathrm{mb}$ for both the experiments on all three days. It is interesting to note that the difference patterns at both levels evolve coherently in experiment-2, while in experiment-1 the upper level displayed a more delayed response to moisture change than the lower level. Yet another interesting feature is the larger differences in circulation in experiment- 2 for comparatively smaller increase in the moisture fields. The reason for this may be attributed to the latent heat release, which increases as the moisture fields reach the saturation level. However in experiment-1, the reduction in moisture does not make a significant change in patterns of latent heat, except for the points where the original values of moisture were near saturation. This way the errors in circulation patterns are more susceptible to the positive errors in the initial moisture fields than the negative errors. On day 3 the wind differences at $850 \mathrm{mb}$ show a remarkable cyclonic loop on the northern side of the nudging domain, while a strong easterly flow develops across the southern half of the domain. Divergence difference fields show that on day 3 the convergence at $850 \mathrm{mb}$ is localized, stronger and shifted northwestward as compared to its day-1 position. On day 3 the formation of a divergence zone south of the initial convergence is also noticed. There is an indication that the strong convergence over the Arabian Sea and lower level westerlies together played the role in increasing moisture at the northeastern part of the experimental domain (figure $3 \mathrm{c}$ and $3 \mathrm{~b}$ ). There seems to be a strong coupling in the form of a positive feedback between the diabatic heating resulting from strong moisture convergence (figure 4) and the low level circulation.

Table 1. Root Mean Square (RMS) wind difference at 850 and $200 \mathrm{mb}$ for Experiment-1 and Experiment- 2 Values in $\mathrm{m} / \mathrm{s}$

\begin{tabular}{lcccc}
\hline Day & \multicolumn{2}{c}{ Exp-1 } & \multicolumn{2}{c}{ Exp-2 } \\
no & $850 \mathrm{mb}$ & $200 \mathrm{mb}$ & $850 \mathrm{mb}$ & $200 \mathrm{mb}$ \\
\hline 1 & 0.50 & 0.50 & 0.74 & 1.71 \\
2 & 1.61 & 2.79 & 2.67 & 5.83 \\
3 & 2.58 & 4.32 & 3.29 & 5.74 \\
\hline
\end{tabular}


Table 2. (a) Moisture flux convergence (negative values indicate convergence while positive values indicate divergence). Values are in $\left(\mathrm{gm} / \mathrm{cm}^{2} / \mathrm{day}\right)$

\begin{tabular}{lccc}
\hline Day & Control & Experiment-1 & Experiment-2 \\
\hline 0 & -0.20 & -0.20 & -0.20 \\
1 & -0.04 & 0.09 & -1.23 \\
2 & -0.65 & 0.25 & -1.74 \\
3 & -0.27 & 0.80 & -0.54 \\
\hline
\end{tabular}

Table 2. (b) Evaporative fluxes ( $\mathrm{gm} / \mathrm{cm}^{2} / \mathrm{day}$ ).

\begin{tabular}{lccc}
\hline Day & Control & Experiment-1 & Experiment-2 \\
\hline 0 & 0.13 & 0.13 & 0.13 \\
1 & 0.28 & 1.62 & 0.27 \\
2 & 0.34 & 0.71 & 0.32 \\
3 & 0.31 & 0.42 & 0.31 \\
\hline
\end{tabular}

\subsection{Evaporation and moisture convergence}

Table 2 and figure 4 show the low level evaporative flux and the vertically integrated moisture flux convergence over the nudging region for three days of integration of the control, experiment-1 and experiment-2. Evaporative fluxes show a sudden increase of $100 \%$ on day- 1 in the control run, which may be attributed to a sharp increase on zonal kinetic energy between 11 June and 12 June 1979 (Sharma and Sadourny 1985). The evaporative flux remains saturated hereafter at around $0.3 \mathrm{gm} / \mathrm{cm}^{2} / \mathrm{day}$ for the next two days. In experiment-1, the dry atmospheric conditions favour evaporation and the flux rises to $1.6 \mathrm{gm} / \mathrm{cm}^{2} /$ day on day 1 , which then came back to $0-48 \mathrm{gm} / \mathrm{cm}^{2} /$ day on day 3 when the forcing terms in the moisture equation are removed. However, increasing the moisture in experiment-2 does not affect the evaporation significantly, mainly because the atmosphere was never allowed to cross the saturation level prescribed by the control run. The moisture flux convergence, unlike the evaporative flux, is a highly variable parameter in control runs and there are diurnal fluctuations. The maximum convergence occurs on day 2 of the control run and it is $0.65 \mathrm{gm} / \mathrm{cm}^{2} /$ day. The effect of negative moisture anomalies is seen as an alteration of these patterns from convergence to divergence, which increases with time and on day 3 it reaches $0.8 \mathrm{gm} / \mathrm{cm}^{2} /$ day. Patterns of flux convergence are consistent with the patterns of circulation difference (figure 5) or divergence difference at the lower level. In experiment- 2 the induction of positive moisture anomalies leads to further convergence of moisture, the maximum of which occurs on day 2 of integration $(1.74 \mathrm{gm} /$ $\mathrm{cm}^{2} /$ day). The values drop to $0.54 \mathrm{gm} / \mathrm{cm}^{2} /$ day on day 3 . It seems that the advection of this extra moisture to the upper levels of northeastern sector of the nudging region by mean zonal and vertical flow leads to the termination of a possible feedback between the diabatic heating and moisture convergence. This phenomenon indicates that a positive feedback between moist processes and dynamics exist as long as the moisture is forced to increase by some mechanism. In the absence of external forcing 
this feedback seems to terminate. The average values of flux convergence in experiment-1 and experiment-2 are quite different from each other on day 3 which is probably a manifestation of the model sensitivity to initial wind fields. In other words, the difference of circulation caused by the anomalies of experiment-1 and experiment-2 keeps growing with time, even if the forcings are withdrawn and the humidity fields have retained their original patterns. This fact stresses the need for accurate wind observation in the initial state of the model.

A close examination of evaporation and convergence patterns for experiment-1 shows the dominance of evaporation on day-1 after which it decreases, while from day 1 onwards the flux convergence begins to grow. In dry atmospheric conditions, evaporation acts at a moisture source in the initial phase (upto one day in experiment-1) after which the convergence and advection take over till saturation takes place.

\subsection{Heating}

The vertical profile across a longitude line of the model derived convective heating rates at $14^{\circ} \mathrm{N}$ (across the centre of the nudging domain) for day-1 is shown in figure 7 . The maximum heating at this latitude occurs around $65^{\circ} \mathrm{E}$ and $\sigma=0.64$ in the control run. $A$ decrease in moisture (experiment-1) reduces the heating rates approximately by a factor of 0.5 . But, the heating patterns are similar in magnitude to their control counterparts. In experiment-2, however, the effect of moisture increase is noticed as the development of a secondary heating maxima, which lies on the eastern side of the primary maxima. The amount of heating increases below the $\sigma=0.64$ level. This
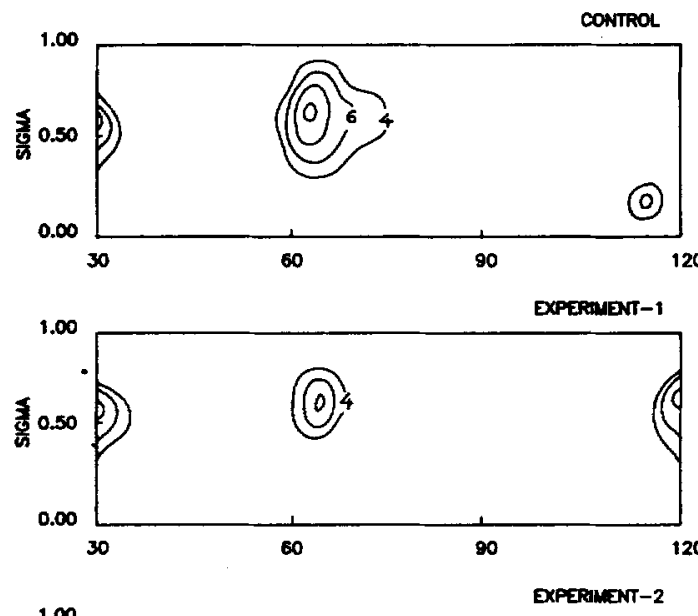

(a)

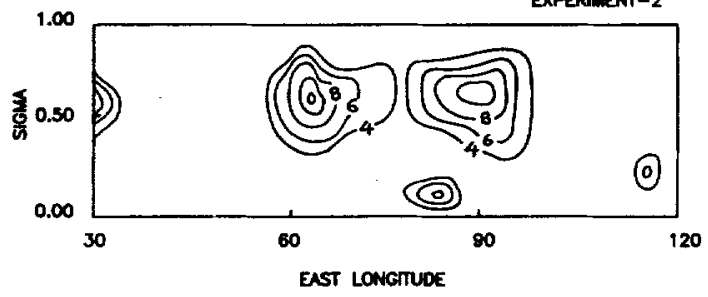

(b)

(c)

Figure 7. Longitude-sigma cross section of absolute heating rates on day-1 (Unit $=K /$ Day) for (a) Control (b) Experiment-1 and (c) Experiment-2. 
indicates the development of a shallow convection in a near saturated atmosphere and their advection by strong low level westerlies.

In both the experiments the difference in divergence or convergence indicates that the centre of the circulation was produced to the north (and slightly west) of the region of maximum moisture anomaly. This coincides with the regions of maximum heating, while a circulation pattern was produced at upper levels, identical but opposite in nature to its low level counterpart. These features bear striking resemblance to the solutions found by Sashegyi and Giesler (1987), and Gill (1980) for the linearised response to localised heat sources situated off the equator. Gill (1980) showed that the southerly flow in the region of heating (as in figure $6 c$ ) is consistent with a linear vorticity balance, in which negative advection of planetary vorticity balances the positive vorticity generated by convergence.

\section{Conclusions}

The main conclusions from this study are as follows:

1. Although the model seems to have 'resilience' for moisture fields, the time taken by the model in normalizing an ill-defined moisture field is approximately 3 days. During this time the moisture fields affect the circulation in a significant way. This stresses the accuracy requirement for the initial moisture field for medium range prediction (for periods 3-5 days).

2. Anomalies produced in the mean monsoon flow due to the variation of humidity fields keep on growing with time at all levels, even after the forcing terms in the moisture equation are withdrawn (table 1). It shows a highly sensitive dependence of wind fields to initial conditions and stresses the need for more accurate observations of wind.

3. Convective heating has an important role in coupling the lower and upper level momentum fields. The time period for coupling 850 and $200 \mathrm{mb}$ is approximately one day for monsoonal systems.

4. Positive anomaly of moisture fields affects the circulation more significantly than a negative anomaly of similar magnitude.

\section{References}

Cadet D 1986 Fluctuations of precipitable water over the Indian Ocean during the 1979 summer monsoon; Tellus A38 170-177

Courtier Philippe and Geleyn Jean-Francois 1988 A global numerical weather prediction with variable resolution: Application to the shallow water equations; Q. J. R. Meteor. Soc. 114 1321-1346

Gill A E 1980 Some simple solutions for heat induced tropical circulations; $Q$. J. R. Meteor. Soc. $106447-462$

Goswami B N and Shukla J 1984 Quasi periodic oscillations in a symmetric general circulation model; $J$. Atmos. Sci. 41 20-37

Hoke J E and Anthes R A 1976 The initialization of numerical models by a dynamic initialization technique; Mon. Weather Rev. 104 1551-1556

Kishtawal C M, Pal P K and Narayanan M S 1991 Water vapour periodicities over Indian Ocean during contrasting monsoons from NOAA satellite data; Proc. Indian Acad. Sci. (Earth Planet. Sci.) 100 341-359

Krishnamurti T N and Kanamitsu M 1973 A study of a coasting easterly wave; Tellus 25 568-586 
Kuo H L 1965 On the formation and intensification of tropical cyclones through latent heat release by cumulus convection; J. Atmos. Sci. 22 40-63

Manabe $S$ and Strickler R F 1965 On the thermal equilibrium of the atmosphere with convective adjustment; J. Atmos. Sci. 21 361-385

Ramamurthy M K and Carr F H 1987 Four dimensional data assimilation in the monsoon region. Part I: Experiment with wind data; Mon. Weather Rev. 115 1678-1706

Sashegyi K D and Geisler J E 1987 A linear model study of cross-equatorial flow forced by summer monsoon heat sources; J. Atmos. Sci. 44 1706-1722

Selby J E A, Shettle E P and McClachey R A 1976 Supplement LOWTRAN 3B. AFGT-76-0258; Envir. Res. Papers. No. 587

Sharma O P and Sadourny R 1985 Numerical experimentation with FGGE data: Simulation of the 1979 monsoon onset using a stretched coordinate version of the LMD-GCM; Report on the seminar on progress in Tropical Meteorology as a result of the Global Weather Experiment, GARP Special Report 44 WMO, Geneva, IV, 78-86

Sharma O P, Upadhyaya H C, Th Braine-Bonnaire and Sadourny R 1986 Experiments on regional forecasting using a stretched coordinate general circulation model; Short and Medium Range Numerical Weather Prediction (ed.) T Matsuno, Met. Soc. Japan

Smagorinsky J, Manabe S and Holloway Leith Jr. 1965 Results from a nine-level general circulation model of the atmosphere; Mon. Weather Rev. 93 727-768

Smagorinsky J, Miyakoda K and Strickler R F 1970 The relative importance of variables in initial conditions for dynamical weather prediction; Tellus 22 121-153 